\title{
Do you feel disempowered? It seems you should be
}

Marmot, [1] in a recent blog for the Health Foundation, addressed the topic of 'Dealing with an Epidemic of Disempowerment'. He suggested that 'world health as measured by life expectancy, is improving'. He also cited the work of Case and Deaton [2] that demonstrates declining mortality rates across a range of developed countries, including Australia. However, those authors also go on to demonstrate that both mortality and morbidity in mid-life, white non-Hispanic Americans are no longer in decline but are increasing in comparison to where they were and with respect to other cultural groups within the United States that continue downward trends. The causes of mortality were said to be poisoning due to drug and alcohol, suicide, liver disease and violent deaths.

In the United Kingdom Marmot demonstrates further disparities in major cities such as Glasgow, Liverpool and Manchester. He cites a disparity in mortality within one Scottish city of 28 years and proposes that the causes of death are the same attributed to those of the white nonHispanic population in the United States.

While Australia can afford to be self-congratulatory at the national data level and in comparison to other OECD countries, there are areas of concern nationally and stark differences in health outcomes are easily observable in rural communities and at the local government (LGA) data level when compared with each other and against State and national level data. A look at available data from primary health network websites shows that observable differences across urban, regional and remote communities are easily detected and some correlation of poor health outcomes with poor socio-economic determinants, together with a shortage of health workforce and other observable access and equity issues, are evident in those contexts. [3]

Nationally, 'Chronic diseases are the leading cause of ill health and death in Australia', [4, p.13] with more than 50\% of Australians having one chronic condition, half of those having more than two such conditions. In addition, more than $5 \%$ of Australians have diabetes. 'People in regional and remote areas are more likely to die prematurely than their major city counterparts'. [4, p.14]
Marmot emphasises 'this mortality crisis is not a medical care issue' because of the nature of the disease group and that 'these causes are substantially psychosocial in origin... in which social conditions affect health and health inequalities.' [4] Marmot goes further by suggesting that we need 'to put health equity at the centre of our activities'... in... 'a practical pursuit of social justice'. [4] In addition to the great disparity of equity in access for communities with poor socio-economic determinants, the increasing burden of chronic disease is often ascribed to the increased ageing population. Sometimes the aged are seen as the problem in these contexts.

However, seeing ageing populations as the problem is not social justice because they, like all other groups, are entitled to equitable access. Ageing populations are the 'new social reality' to which we are all urged to respond creatively in a society where the 'elderly are enabled to remain meaningful participants in the community'. [5, pp. 337-338] The purpose of public policy, and the role of providers and health professionals and the services they deliver should not be to 'marginalize and disempower' older people by the way we have developed and/or purchased'the very programs meant to "help" them'. [5, p.339] So, irrespective of being elderly or coming from a background that lacks opportunities for education, employment and social inclusion, social justice suggests that they are 'persons who continue to desire dignified inclusion in the community'. [5, p.341]

Historically in Australia communities established local hospitals, aged care facilities and other community-based services. At the time, this is said to have occurred with little interest from government at any level until there was a growing awareness that the uncontrolled development of these sectors by communities had longer-term implications for government capital and operating expenditure into the future. [5]

So consequently, instead of having standalone community controlled public hospitals we have large systemic bureaucracies running multiple hospitals and health services as part of a system-wide approach to healthcare. These 
organisations are substantially creatures of the respective state governments delivering service to a geographic region with the word 'local' in the organisational title. In aged and community care, aggregation and centralisation have also occurred with many, but not all, of the large organisations still of a 'not for profit' and/or faith-based status that are state or nationally organised.

In the Primary Healthcare (PHC) sector the delivery of healthcare is still substantially fragmented with individually owned general practice and an immense array of local and national service providers. Without much debate or the provision of coherent public policy and, in what might be described as undue haste to dismantle the coordinating and supportive roles of Medicare Locals, primary health networks (PHNs) were established.

PHNs were established some two years ago with a major responsibility to introduce commissioning as a concept in funding the primary care sector. Hence part of their role was based on establishing a 'quasi market' in PHC. The community care market at the state government level had already gone down that path with a departure from delivery by state government agencies to that delivered by the 'third sector'. The third sector being that outside of direct government public sector control and outside the 'for profit' private sector and consisting mostly of NGOs, not for profit, charitable and faith-based organisations that are increasingly nationally organised.

So the point of this evolutionary change in service delivery from the state to the private and third sector has been fostered by governments of all persuasions, for apparent purposes of creating greater efficiency and purchasing power and rationalising the degree of provision. Relationships before this evolution to markets were and still are complex mostly because of the contradictions a Federation of States and Territories brings. However, before these changes it has been said that there was a greater degree of'communication, collaboration and cooperation to ensure people could access the best combination of services possible.' [6, p.43]

The consequences of the quasi market approach and national competition policy are that those in need of care are minutely assessed without any guarantee of access to services. Outside of capital cities and urban centres there may be few or only one provider yet the process of testing the market is applied and the outcome may well mean a move away from the one local provider to a distantly based national provider who has no investment in a local community and no or little investment at the management and professional level, in the social capital of those communities. In fact, the staff of the once viable local provider run the risk of having to move their employment or move from the town. Contracted and independent practising health professionals may find themselves re-contracting to differing providers on an annual basis to deliver the same services to the same clients, and the clients must adjust to these differences regularly! Many of these occurrences correctly forecast a decade ago are now the context in which we work. [6] They also present a level of concern about risk management where the provider and professional staff are few and far between.

This move to centralised control, management and delivery of the vast array of health and community sector services also adversely impacts on the social capital of local communities. The intellectual contributions of health and community professionals and senior managers are lost to those communities, reducing the leadership and support to those marginalised groups that remain in place. The literature also suggests a strong relationship between social engagement and social networks. [6, p.37] Social capital 'reflects the collective benefits of community engagement'... and it 'depends on people's involvement, all forms of social exclusion damage a community's potential store of social capital'. [6, p. 37]

The reduction or retraction of services, along with the relocation of senior managers and professionally trained disciplines to distant and larger centres further contributes to the decline of rural towns and reduces their capacity to respond to socio-economic determinants that contribute to poorer health outcomes, morbidity and mortality. The very thing that the intended services are meant to address!

It will require a greater consciousness to emerge from our collective politicians, policymakers, health professionals, bureaucrats and communities to address the challenges described in this editorial. The National Rural Health Alliance (NRHA) has a national strategy in place. [7] The Federal government has delivered PHNs and a promise of a Rural Health Commissioner and the concept of 'Health Care Homes. However, you do not need to read the compelling statistics carefully provided in 'fact sheets' by the NRHA to understand the concepts of poverty in rural Australia, the poorer outcomes for rural dwellers or the inequity of both funding and access to care. [8] Just ask any group of rural citizens, ask the local Mayor or Council General Manager, as this Editor does, living in a regional community, and they will talk to you about the difficulty of financial and physical 
access to healthcare. It seems that the further away you live from urban areas the poorer the access to care and improved health outcomes.

I am mindful that we are a highly developed nation but with significant populations of disadvantage. Perhaps we should look to the success of other countries that have emerged and or are emerging from an impoverished status of the third world to see how they have handled disempowerment. Remember we are talking about obesity, diabetes and inactivity, poor diet, low birth weights, alcohol, drug and cigarette addiction, suicide and mental health aberrations. These are increasingly the challenges of those countries that are becoming increasingly urbanised. Remember that Marmot [1] defines the challenge as one not being necessarily addressed by medical intervention but mostly located in the psychosocial sphere that might be more adequately addressed by social engagement and social movements.

There are many examples in developing countries of services being delivered by or accessed through a defined role of 'barefoot doctor', village health volunteer or community village worker to name a few. These are citizens trained at the public health intervention level to detect need, screen, provide an access point at village community level to appropriate and often more westernised services at the higher level and to convince villagers to access health services at the PHC level.

These countries have in common a sense of the extended family and a respect for culture that includes an emotional and social context within which relationships and care are provided. This is also the case in Australia where Aboriginal and Torres Strait Islander populations place great emphasis on culture, country and community underpinned by social and emotional support. There is emerging interest in the extension of the Aboriginal health worker/practitioner role within general practice as a counterbalance to the lack of timely access to referred psychological and counselling services. It could also be valuable in a social movement role to mobilise the wider communities with poor health outcomes. This initiative alone has merit and would help to both maintain communities and a scarce workforce.

Secondly, countries like Thailand are researching how best to improve care at the local community level through district health services. As a starting point, they want to take an across-sector approach that engages communities, education, local government and health working together to improve access and equity for healthcare. This means at the district level Thailand is trying to encourage all sectors including public, private, local government and community to work together to integrate health and social care for its population in order to lift up their quality of life. This approach also goes to the mandates of the public sector agencies and Ministries collectively. This sounds like an approach that should advantage rural communities.

They understand empowerment. They have also been reformist in achieving Millennium Development Goals (MDGs) and more recently working towards Sustainable Development Goals (SDGs) to demonstrate improved health outcomes. SDG 3, in particular asks us to 'Ensure healthy lives and promote well-being for all ages.' [9] There is little discussion or profile around these United Nation objectives in the Australian context. If they were brought to the centre stage of policymaking they might help create social movement in communities and encourage those sectors to come together in some form of community consortium to do things differently and more effectively, like they are doing in Thailand.

In a recent editorial I emphasised the need for a change of culture from valuing healthcare to one that values health. That editorial also suggested that we must learn to work across sectors. The health sector needs to engage the other traditional sectors to improve health outcomes that are essentially the result of poor access to education, employment and social engagement. [10] Like the historical context, where communities started their own hospitals and aged care facilities, they may have to reinvent that enthusiasm by creating social movement within their communities to more effectively address areas of poor socio-economic determinants in more creative ways.

Can we learn from developing and lower middle-income countries that have been more astute, strategic and engaging than Australia? Are we capable of recognising and moving towards empowering communities in this respect? Can we ensure that in implementing government policy we do not further impoverish the social capital of communities particularly those in rural locations?

\section{DS Briggs}

Editor 


\section{References}

1. Marmot, Sir Michael. 2017. Dealing with an epidemic of disempowerment. The Health Foundation; June 292017. Available from: http://www.health.org.uk//blog/dealing-epidemicdisempowerment.

2. Case A, Deaton A. Rising morbidity and mortality in midlife among white non Hispanic Americans in the 21st century. Proceedings of the National Academy of Sciences of the United States of America. PNAS. December 8, 2015;(112)49. Available from: http://www.pnas.org/content/112/49/15078.full.

3. HNECCPHN. Health Planning Compass 2016. Hunter New England Central Coast Primary Health Network. Available from: http:// www.hneccphn.com.au/media/3824/compass-jun-16-finalcompressed.pdf.

4. AlHW. 2016 Australia's health 2016. Australia's health series no.15 Cat. No. 199. Canberra: Australian Institute of Health and Welfare. Available from: http://www.aihw.gov.au/publicationdetail/?id=60129555544.

5. Crichton A. Slowly taking control? Australian governments and health care provision 1788-1988. North Sydney: Allen \& Unwin; 1990.

6. Leonard R, Johansson S. Policies and practices relating to the active engagement of older people in the community: a comparison of Sweden and Australia. Int J Soc Welfare. 2008: 17:37-45.

7. National Rural Health Alliance. (NRHA) 2017. Knowing the issues: new and old Issues in rural and remote health. Available from: http://ruralhealth.org.au/advocacy/knowing-the-issues.

8. National Rural Health Alliance. (NRHA) 2017. Fact Sheets. Available from: http://ruralhealth.org.au/factsheets/thumbs.

9. Tejativaddhana P, Briggs DS, Thonglor R. From global to local: strengthening district health systems management as entry point to achieve health-related sustainable development goals. Asia Pac J Health Manag. 2016;11(3):81-86.

10. Briggs DS. Building a culture of health. Asia Pac J Health Manag. 2017; 12(1):6-8. 